la revue La revue pour l'histoire du CNRS

POUR LHISTORE DU CNRS $10 \mid 2004$

Penser la pensée. Les sciences cognitives

\title{
La régionalisation : rapprocher la recherche des réalités régionales
}

\section{Girolamo Ramunni}

\section{(2) OpenEdition \\ 1 Journals}

Édition électronique

URL : https://journals.openedition.org/histoire-cnrs/592

DOI : $10.4000 /$ histoire-cnrs. 592

ISSN : 1955-2408

Éditeur

CNRS Éditions

Édition imprimée

Date de publication : 5 mai 2004

ISBN : 978-2-271-06145-4

ISSN : $1298-9800$

\section{Référence électronique}

Girolamo Ramunni, «La régionalisation : rapprocher la recherche des réalités régionales », La revue pour l'histoire du CNRS [En ligne], 10 | 2004, mis en ligne le 03 novembre 2006, consulté le 20 mai 2021. URL : http://journals.openedition.org/histoire-cnrs/592 ; DOI : https://doi.org/10.4000/histoire-cnrs. 592

Ce document a été généré automatiquement le 20 mai 2021.

Comité pour I'histoire du CNRS 


\title{
La régionalisation : rapprocher la recherche des réalités régionales
}

\author{
Girolamo Ramunni
}

En juin 2004, un laboratoire mixte CNRS-Rhodia ouvrira ses portes à Pessac en Gironde. Quarante chercheurs, dont quinze recrutés sur de nouveaux postes chez Rhodia et six au CNRS, auxquels s'ajouteront des thésards, des post-doc., des chercheurs associés et/ ou de passage, seront réunis en vue de réduire les délais de production, donc de mise sur le marché, de nouveaux produits et optimiser les procédés de fabrication. Ce « laboratoire du futur " permettra à des chercheurs spécialistes de physico-chimie, chimie des procédés, nanotechnologies, électronique, informatique et mathématiques appliquées de travailler ensemble ${ }^{1}$. Cet accord intervient presque trente ans après un premier accord entre le CNRS et un groupe chimique français, Rhône-Poulenc (en août 1975), dont Rhodia faisait d'ailleurs alors partie. Les objectifs et le contexte sont cependant radicalement différents. En 1975, il s'agissait avant tout d'établir un dialogue entre chercheurs CNRS et chercheurs industriels, éventuellement de développer quelques travaux en commun. Ce premier accord-cadre était signé dans le contexte d'une régionalisation encore en phase expérimentale. On parlait beaucoup à l'époque de délocalisation des structures de recherche en province. En 1975, alors que les régions n'existaient pas encore en tant qu'entités politiques et administratives, il s'agissait donc de mener une expérience pilote. La délocalisation en région était alors pensée aussi comme un moyen de rapprocher la recherche académique des réalités industrielles locales.

Une expérience pilote

2 En 1972, une première expérience pilote de régionalisation de la recherche est tentée en Rhône-Alpes. François Juillet, délégué régional de la DGRST, est nommé auprès du préfet de région. Il se souvient ${ }^{2}$ : «En 1972, à la demande de Pierre Aigrain, en accord avec Hubert Curien et Bernard Grégory, j'ai commencé une expérience de régionalisation de la recherche ; Pierre Aigrain souhaitait qu'une expérience soit réalisée sur un territoire équivalant à une région allemande ou européenne moyenne. D’où l'idée de mener cette expérience en regroupant les régions Rhône-Alpes, Auvergne 
et Bourgogne. "S'agissant d'une mission exploratoire, le délégué avait l'avantage de pouvoir prendre des initiatives, en en référant directement aux directeurs de la DGRST et du CNRS, son action étant évaluée a posteriori. Des décisions pouvaient ainsi être prises rapidement. Sa position administrative donnait au délégué une fonction de coordonnateur entre les divers centres de recherche locaux, indépendamment des organismes de recherche ou des ministères de rattachement. Cette expérience de coordination locale a été poussée jusqu'à faire gérer une action concertée DGRST en province. L'action concertée " matériaux composites de grande diffusion " a été en effet gérée à Lyon.

Une des premières phases de la coordination a été de promouvoir l'échange entre les acteurs locaux, de créer des lieux de rencontre. Cela passait par la création de groupes thématiques. L'un d'entre eux, par exemple, a été appelé G3F, qui voulait dire «fibres, feuilles, films » et regroupait le Centre technique du papier, celui du cuir et l'Institut textile de France. Cette collaboration entre trois centres techniques a conduit au développement de réunions de concertation des centres techniques installés dans la région, ce qui a permis non seulement de se connaitre mais aussi, le cas échéant, de faire des économies en mettant en commun des moyens lourds. L'interlocuteur naturel, quand il s'agissait de créer de nouvelles structures ou d'accueillir des laboratoires venant de la région parisienne, était l'Établissement public régional, ancêtre des actuelles régions. En utilisant les faibles crédits de l'époque (180 000 francs), il a été possible de " délocaliser » certains laboratoires CNRS, comme celui de microanalyse, qui a été installé à Solèze. Ce premier centre est devenu le noyau du développement du pôle chimie en région, avec l'installation de l'Institut français du pétrole (IFP) et la création du Laboratoire de matière organique (LMO).

La souplesse découlant du statut et de la structure de mission, donc l'absence d'une organisation administrative contraignante, comme ce sera le cas plus tard avec l'institutionnalisation de la fonction de délégué régional à la recherche, permettait de trouver rapidement des solutions selon les problèmes posés. Un exemple de cette souplesse tient dans l'action menée en faveur de l'industrie du papier qui, au milieu des années 1970, était en pleine crise. François Juillet ${ }^{3}$ l'explique ainsi : «Pierre Cognard, directeur du Centre technique du papier, a proposé de trouver des financements pour développer des recherches tendant à résoudre les problèmes rencontrés par cette industrie. Il s'agissait, en guise de défi, de financer les cent premières suggestions provenant des industriels. Une fois la liste arrêtée, les entreprises ont été visitées par un jeune ingénieur des Mines. L'objectif était de traduire, si possible, les difficultés rencontrées par les industriels en problématiques de recherche. Cette simple traduction a permis de constater que pas moins de $80 \%$ des problèmes étaient de fait déjà résolus par la recherche académique. On connaissait déjà les solutions à adopter. Mais le langage des chercheurs pour les exprimer était un obstacle. En résumé, il s'agissait d'une barrière linguistique qui empêchait les chercheurs et les industriels de communiquer entre eux. D'où l'idée d'organiser des journées pendant lesquelles chercheurs et industriels s'efforçaient de dialoguer, dans un langage qui ne faisait plus appel au "jargon", sur les problèmes et leurs solutions. Il a ainsi été possible de repérer des pistes de recherche et de mobiliser les chercheurs sur des points où l'industrie rencontrait des difficultés; les recherches s'y rapportant ont été soutenues par une action thématique programmée (ATP) cofinancée par le CNRS et la DGRST. L'un des résultats de cette action a été le développement de l'École de papeterie, signe que la demande industrielle était forte à nouveau. Ce qui a été possible pour le papier ne l'a 
cependant pas été pour le textile - il ne s'agit pas là d'une recette miracle -, car l'Institut français du textile était structuré en plusieurs centres, dont Paris, Lyon et Troyes. Cette structure multiple empêchait d'atteindre la masse critique de chercheurs permettant de réaliser une telle structuration qui, pour être efficace, doit pouvoir se dérouler sur un temps relativement long."

5 Les centres de recherche technique avaient été créés comme des structures d'aide à l'industrie par branches professionnelles. Déjà à la Libération, la question de la coordination entre ces centres s'était posée au niveau national. Le gouvernement avait proposé en 1945 la création d'une structure fédérative, un institut permettant de rassembler tous ces centres. Sa réalisation avait rencontré l'opposition ferme de certains centres techniques, comme ceux de l'horlogerie ou du machinisme agricole, qui voulaient garder jalousement leur autonomie. Individuellement, ces centres prenaient au coup par coup des contacts avec des laboratoires académiques, quand des problèmes les dépassant se posaient. La coordination et un dialogue suivi étaient donc un progrès considérable.

6 En l'absence de ces structures professionnelles, force était de dialoguer directement avec l'industrie, comme cela a été le cas de Rhône-Poulenc, ainsi que le décrit François Juillet $^{4}:$ «On avait constaté pendant des réunions auxquelles participait André Pacoud, membre du Comité exécutif et directeur des recherches et du développement chez Rhône-Poulenc, qu'il y avait des thématiques de recherche qui étaient développées de manière indépendante au CNRS et dans l'industrie. D'où l'idée de mener quelques recherches en commun, en vue de tester une collaboration plus étendue. Bernard Grégory, directeur général du CNRS, informé de ces coopérations ponctuelles a non seulement donné son accord, mais il a demandé qu'elles soient renforcées. René Gillet, président de Rhône-Poulenc, informé par André Pacoud, a donné lui aussi son accord. Ce qui a débouché, en août 1975, sur l'accord entre le CNRS et Rhône-Poulenc. »

7 Cet accord prévoyait une concertation entre chercheurs appartenant aux deux structures plus qu'une réelle conduction de travaux de recherche en commun ou la création de structures de recherche communes. Ce qui a semblé aux syndicats contrevenir à l'éthique du chercheur académique a été la question de la confidentialité des résultats, soit obtenus, soit connus. La clause de confidentialité était valable pour quinze ans. Les tracts syndicaux de l'époque mettent en évidence cette clause comme une entorse grave remettant en cause l'éthique de la recherche publique. La suspicion vis-à-vis de cet accord était augmentée par la décision de la direction du CNRS de ne pas communiquer le texte de la convention aux syndicats. Ce texte, tout comme les réactions, positives et négatives, montre un problème qui s'était déjà posé ailleurs qu'en France, en particulier en ce qui concerne l'origine des ordinateurs ou la maîtrise, civile comme militaire, de l'énergie atomique ${ }^{5}$. Il y a des moments où les deux logiques, la poursuite de la recherche académique et le développement des recherches à finalité industrielle, peuvent entrer en conflit. Ce qui ne doit pas interdire a priori toute collaboration.

Un autre aspect, étroitement lié au premier, était la crise de l'image de la structure de la recherche qui, depuis la fin des années 1960, s'était développée en France à la suite de séminaires de réflexion auxquels avaient participé les responsables du CNRS. La vision habituelle de la recherche structurée en deux branches - la recherche pure, ou fondamentale, ou académique et la recherche appliquée - commençait à être considérée comme une image simpliste des multiples activités qui sont rassemblées 
sous le chapitre de la recherche. Ces changements marquent une réelle rupture qui, même si les signes avant-coureurs, en France, avaient été masqués par l'exceptionnelle expansion de la recherche dans les années 1960, montre que la vision de la recherche héritée du xIX ${ }^{e}$ siècle et encore dominante en France avait du mal à être abandonnée même si elle ne correspondait plus à l'organisation du $\mathrm{xx}^{\mathrm{e}}$ siècle finissant ${ }^{6}$.

À ces éléments « internes » à la communauté des chercheurs s'ajoutait comme élément nouveau un aspect plus politique : la volonté de régionalisation de la recherche souhaitée par les responsables politiques et acceptée par les dirigeants des organismes de recherche publics.

10 L'accord-cadre avec Rhône-Poulenc est indiqué par la direction du CNRS comme l'une des réalisations de sa politique " de relations avec le monde socio-économique, relations fondées sur la recherche de l'ouverture et sur la défense des intérêts légitimes de la recherche et des chercheurs ${ }^{7}$ ». En effet, l'année 1975 est présentée par les responsables des diverses institutions en charge du développement de la recherche en France, comme une année cruciale dans l'évolution et l'expansion de la place de la recherche dans l'économie du pays. On est, rappelons-le, dans un climat de crise consécutif au choc pétrolier. Deux conseils interministériels, les 28 février et 3 novembre 1975, ont défini, pour le premier, les grandes orientations de la recherche, pour le deuxième, les modifications de structure des organismes en place. Après la décision concernant le programme électronucléaire français, décidé sous la présidence de Georges Pompidou, deux nouveaux projets nationaux sont arrêtés sous Valéry Giscard d'Estaing : la relance de l'industrie informatique et le train à très grande vitesse (TGV). En février 1975, c'est la recherche agronomique et industrielle au sens large qui constitue la priorité. Ceci est décidé dans la phase des travaux préparatoires pour le $\mathrm{VII}^{e}$ Plan, et signifie l'engagement de l'État sur plusieurs années à apporter un soutien constant à l'expansion de la recherche. L'augmentation régulière des crédits affectés à la recherche ainsi qu'une croissance constante de l'emploi scientifique devaient être précédées d'une "étude sur l'efficacité de la recherche sous sa forme actuelle, notamment dans les universités et au CNRS ${ }^{8} »$. Pour le CNRS qui, d'après les statistiques du secrétariat d'État aux Universités, représente environ 80 \% de la recherche de base en France, ces décisions auraient permis de rompre avec la récession des crédits et des postes qui caractérisait la période post-1968. La décision du CNRS de construire le grand accélérateur national à ions lourds (Ganil) à Caen marque la volonté de redémarrer la construction d'équipements lourds nécessaires à cette recherche de base. Le CNRS procède par ailleurs le 2 novembre 1975 à une modification de son organigramme.

11 Le maitre mot de la politique de soutien à la recherche est la coordination. Ce qui conduit à une définition des rôles respectifs de l'Université et du CNRS. On attribuait habituellement à l'Université le rôle de formation et au CNRS celui de soutien à la production des connaissances ; la politique des équipes associées, développée par le CNRS sous la direction de Pierre Jacquinot, devait répondre à la nécessité de l'implantation régionale de la recherche sans nuire à une vision nationale que seul le CNRS pouvait avoir. D'où le rôle d'évaluateur, mais aussi de promoteur de la recherche pluridisciplinaire dévolu au CNRS. Bref, il fallait avant tout veiller à éviter le gaspillage et notamment les doublons inutiles. La mise en place d'une Mission de coordination de la recherche, placée sous la direction du conseiller scientifique auprès du secrétariat d'État aux Universités, était envisagée. L'objectif recherché était la coordination entre 
la Direction des enseignements supérieurs et de la recherche et le CNRS, pour évaluer la conformité des actions entreprises « à la politique nationale de recherche, à seule fin d'atteindre les objectifs du Plan ${ }^{9}$ ». Le CNRS mesura alors les conséquences, d'une part, de l'octroi en 1959 du premier statut des chercheurs leur assurant la possibilité d'une carrière au CNRS et, d'autre part, de la forte expansion de la recherche dans les années 1960. Il se trouvait à gérer une masse salariale de plus en plus importante, composée de chercheurs et d'ingénieurs, techniciens et administratifs (ITA), auxquels s'ajoutait un nombre élevé de « hors-statut » qui avaient été payés, plusieurs années durant, sur des contrats de recherche. La création du département des sciences physiques pour l'ingénieur (SPI) et la mise en place du Programme interdisciplinaire de recherche sur le développement de l'énergie solaire (Pirdes), dont la direction fut confiée à Robert Chabbal, ancien directeur scientifique pour la physique, marquèrent une restructuration de la direction du Centre. Le directeur de l'Institut national d'astronomie et de géophysique (Inag) devint directeur scientifique pour l'ensemble des sciences de la Terre et de l'espace. De même pour le directeur de l'Institut national de physique nucléaire et de physique des particules (IN2P3). Dès lors, les nominations et avancements des chercheurs sont décidés par la direction du CNRS et les budgets des instituts approuvés par le conseil d'administration du CNRS. La figure du directeur administratif et financier disparaissait, remplacée par celle d'un directeur général adjoint, plus particulièrement chargé des questions administratives et financières et placé sous l'autorité du directeur général ${ }^{10}$. D'autres changements sont introduits qui concernent la carrière des chercheurs et la période de préparation à la recherche par la création des allocations de recherche pour les thésards. Concernant les recrutements, il a été alors décidé par le CNRS de flécher les postes mis au concours.

12 Ces décisions ont une valeur symbolique forte. On a ainsi souligné l'unité du CNRS, écartant la solution envisagée de faire du CNRS " une holding supervisant des organismes sectoriels autonomes ${ }^{11}$ ». L'unité préservée de l'organisme a comme contrepartie la décentralisation, dans le cadre de laquelle les délégations régionales deviennent des lieux de gestion administrative des laboratoires. Entreprise en 1973, cette mise en place était programmée pour s'achever en 1976. La modernisation de l'administration par le développement du « télétraitement informatique reliant au calculateur central la totalité des ordinateurs implantés au sein des administrations déléguées $^{12}$ » est alors indiquée comme une priorité permettant de concilier une gestion locale, au plus près des laboratoires, et une vision globale indispensable à la direction du CNRS pour mener une politique efficace. Pour marquer l'importance de cette régionalisation, il fut décidé que les services de l'administration centrale euxmêmes seraient gérés par une administration déléguée. Le CNRS se conforme ainsi à la politique de décentralisation commencée au début des années 1970 et posée comme condition nécessaire du développement de la recherche dans le VII ${ }^{e}$ Plan.

Le Plan et la régionalisation

La commission de la recherche pour la préparation du VII ${ }^{e}$ Plan comportait en effet un groupe travaillant sur la politique de localisation de la recherche. Toutes les statistiques prouvent alors le rôle prépondérant de la région parisienne, qui représente « 57,6\% de l'effectif des chercheurs rémunérés sur crédits de l'enveloppe recherche, pour seulement $36,4 \%$ des effectifs de l'enseignement supérieur ${ }^{13}$ ». L'objectif posé dans le cadre du VII ${ }^{\mathrm{e}}$ Plan est ambitieux : faire tendre, d'ici 1980, la part des postes nouveaux créés en province vers $75 \%{ }^{14}$; amener le taux de progression annuel des effectifs à $3 \%$ par an ${ }^{15}$. Cela laissait prévoir la création de 7250 emplois pour la période 
du VII Plan (1976-1980) dont environ 5400 en province. Cette prévision ne permettait cependant pas d'atteindre l'objectif du rééquilibrage ( $50 \%$ de l'effectif chercheurs et ITA localisé en dehors de Paris). Il fallait par conséquent prévoir des opérations de décentralisation concernant environ 1600 personnes. Ce calcul fut effectué en ne tenant pas compte du nécessaire renouvellement et rajeunissement des équipes parisiennes. Ce qui augmentait par conséquent le nombre des personnes qu'il fallait convaincre de quitter la région parisienne.

Pour la commission du Plan, le fer de lance de la délocalisation est représenté par les universités, car elles ont de fait une implantation régionale forte. Si l'on pouvait s'appuyer sur le réseau des universités, il fallait néanmoins poser comme condition préalable un changement dans leur politique. Les universités sont en effet contraintes, pour les besoins de l'enseignement, de développer des cours dans de très nombreuses disciplines ; conséquemment, elles ont tendance à développer aussi de la recherche dans toutes les disciplines. Celle-ci est-elle de qualité partout? La réponse à cette question ne fait pas de doute. La seule stratégie possible était que les universités définissent de manière sélective des axes de recherche destinés à favoriser l'éclosion de centres d'excellence. En principe, la Conférence des présidents d'université a donné son accord. Ce qui signifiait aussi une politique de recrutement et d'avancement favorisant la promotion de « la recherche en équipe, sur programme et pluridisciplinaire, et que les attributions de postes prennent mieux en considération la politique scientifique définie par l'Université ${ }^{16}$ ". Il était même suggéré la création de postes, sur le budget de l'enveloppe recherche, réservés aux enseignants-chercheurs souhaitant se consacrer à temps plein à la recherche pendant une période limitée. Si le développement d'une recherche de qualité au sein des universités constituait une priorité, cela ne devait pas conduire à la création à l'Université d'un corps de chercheurs parallèle à celui du CNRS. La délocalisation des grands établissements relevant du secrétariat d'État aux Universités, comme le Collège de France, l'École des hautes études en sciences sociales, l'École pratique des hautes études devait aussi être favorisée. Les centres techniques professionnels, dont la grande majorité était localisée en région parisienne, devaient eux aussi suivre le déplacement des industries en province.

15 Quant au CNRS, « la récente réforme du CNRS mettant en place une décentralisation sur le plan administratif au profit d'administrateurs délégués locaux est un pas important vers une meilleure intégration régionale, et de ce point de vue, constitue une expérience du plus grand intérêt ${ }^{17}$ ». Les autres organismes nationaux de recherche étaient invités à suivre cet exemple. Parmi les actions envisagées dans le rapport du groupe «Politique de localisation de la recherche » de la commission préparatoire du VII Plan, il était prévu la création par le CNRS d'une section géophysique minière à Orléans à partir d'une équipe Inag-CNRS de Garchisy, ainsi que la promotion autour du four d'Odeillo de la recherche en énergie solaire. Il est dit dans ce rapport que le CNRS a déjà procédé de sa propre initiative à la décentralisation dans le domaine de la biologie. Le groupe souhaitait aussi que le CNRS joue un rôle actif dans la politique de délocalisation dans le secteur des sciences de l'homme et de la société.

Du 4 au 6 mai 1977 se réunit à Nice un séminaire sur les technologies et le développement régional, organisé par la Délégation à l'aménagement du territoire, en collaboration avec le ministère de l'Industrie, du Commerce et de l'Artisanat, la DGRST et la Chambre de commerce et d'industrie de Nice et des Alpes-Maritimes. Quatre 
rapports ont été demandés à des institutions universitaires en guise d'introduction aux débats qui se sont déroulés au sein de quatre groupes de travail.

L'attention est portée sur l'industrie en mettant en valeur le savoir-faire par rapport au savoir scientifique, en indiquant l'importance de la technologie du quotidien, souvent oubliée par l'attention prioritaire accordée à la technologie de pointe, et les différences entre petites et moyennes entreprises (PMI) et grande industrie, celle-ci étant partiellement indépendante des ressources régionales alors que les autres identifient leur avenir à celui de la région. On a peu parlé du rôle des organismes de recherche, sauf pour soutenir le programme de délocalisation ou pour souhaiter que des ATP ciblées vers les problèmes des industries régionales soient prévues parmi les actions de soutien et d'orientation de la recherche des grands organismes de recherche. L'importance accordée à la thématique de la région est prouvée par les interventions du secrétaire d'État auprès du Premier ministre chargé de la recherche, Jacques Sourdille, et par le ministre de l'Équipement et de l'Aménagement du territoire, JeanPierre Fourcade. La lecture des communications et des résumés des débats montre que le même changement qui avait été à l'origine des réformes de structure du CNRS conduisait à une analyse du rapport recherche-industrie différent de celui dominant dans les années 1960. En d'autres termes, la régionalisation n'est pas seulement l'occasion de discuter d'aménagement du territoire, mais aussi de modifier les analyses classiques quant à la définition d'une politique de la recherche, l'importance de la recherche pour l'innovation technique et la place cruciale de la recherche et développement, la recherche industrielle et la possibilité pour les PMI d'avoir accès aux sources de l'innovation. D'où l'insistance sur la technologie comme levier de modernisation. De ce fait, la Délégation à l'aménagement du territoire et à l'action régionale (Datar) est devenue un interlocuteur des organismes de recherche. Au cours de ce colloque a été annoncée la création d'un fonds d'aide à la décentralisation de la recherche, cofinancé par la Datar et le secrétariat d'État à la Recherche, avec pour objectif de prendre à sa charge certains surcoûts d'opérations de décentralisation. Tous les projets sont construits autour d'une priorité : la régionalisation doit aussi servir à insérer les laboratoires de la recherche académique, essentiellement de la recherche publique, dans le réseau de l'industrie régionale.

À la fin des années 1970, le bilan des actions de régionalisation est contrasté. Certes, les points positifs ne manquent pas, comme la construction du Ganil à Caen avec le soutien des structures régionales, l'archéologie en Rhône-Alpes, et l'Institut de biologie cellulaire et moléculaire à Nantes. Il restait à généraliser l'expérience des chargés de mission en région, placés sous l'autorité des préfets et travaillant en liaison étroite avec la DGRST. Cela paraissait indispensable du fait du besoin de coordination qui se faisait toujours plus sentir au niveau régional.

Au CNRS, l'expérience des administrations déléguées fut jugée globalement positive. Un pas supplémentaire devait être franchi pour aller vers une certaine normalisation des actions entreprises et des modalités d'action dans les différentes régions. Surtout, il ne fallait pas que les nouvelles structures viennent alourdir l'administration des laboratoires, alors que l'objectif recherché était l'exact opposé. L'informatisation du traitement des opérations, comptables notamment, pour une meilleure utilisation des crédits, sans reports trop importants, semblait constituer un réel défi aux rapporteurs de l'Inspection générale de l'administration ${ }^{18}$ chargés par le secrétaire d'État aux Universités d'évaluer le fonctionnement des administrations déléguées. Au-delà des 
remarques ponctuelles sur le fonctionnement de telle ou de telle autre action qui leur avait été confiée, la conclusion portait sur l'organisation même du CNRS. Les inspecteurs se demandaient si la déconcentration d'un organisme qui avait atteint des dimensions importantes n'était pas trop timide pour avoir des effets réels sur l'implantation régionale de la recherche. Ne fallait-il pas miser sur une réelle gestion régionale de la recherche et laisser au niveau central l'élaboration de la politique de recherche sur le plan national ? Pour diriger les priorités au niveau régional, la direction du CNRS aurait eu comme moyens l'attribution des emplois, des crédits et des équipements. Dans les régions, la responsabilité de l'exécution de cette politique aurait été confiée à une cellule administrative constituée par un organisme collégial de chercheurs, présidé par un chercheur et par un administrateur nommé à Paris ${ }^{19}$. Le réseau informatique aurait permis à l'administration parisienne d'être tenue constamment au courant du suivi des décisions sur le plan local. "L'Inspection s'accorde à penser qu'une déconcentration plus poussée dans la gestion du CNRS dont la contrepartie résiderait dans l'institution d'un contrôle a posteriori répondrait certainement mieux aux dimensions actuelles de cet établissement et à ses perspectives d'évolution ${ }^{20}$. » Dans cette perspective, des projets de réforme ont commencé à circuler à la fin des années 1970, proposant une décentralisation plus poussée pour atteindre les objectifs de simplicité, de souplesse et de rapidité qui auraient dû caractériser le fonctionnement de l'administration du CNRS. Ce n'était pas la compétence des agents du Centre qui était mise en cause mais l'accumulation de règles et de contraintes qui s'était faite progressivement, aux dépens de la rapidité des décisions. L'ambition au début des années 1980 était forte : il s'agissait de créer un fonctionnement souple.

L'élection à la présidence de la République de François Mitterrand conduira à la mise en avant d'autres priorités, dont celle du nouveau statut pour les chercheurs. La première loi Defferre ${ }^{21}$ sur la décentralisation posa à nouveau, mais dans un contexte différent, la question de la régionalisation. Celle-ci, tout comme le développement des liens entre la recherche publique et la recherche industrielle, reste encore un sujet d'actualité, comme le prouve le récent accord du CNRS avec Rhodia.

Thèmes des rapports préliminaires au séminaire « Technologies et développement régional*»

- La formation de complexes technologiques : les enseignements que l'on peut tirer de l'expérience nord-américaine et européenne, par le Center for Policy Alternative du Massachusetts Institute of Technology.

- L'évolution technologique et la firme traditionnelle : les implications pour la politique gouvernementale, par le même centre de recherche américain que précédemment.

- Le rôle de la recherche et des industries à haute technologie dans le développement de la région grenobloise, par l'Institut de recherche et de planification de l'université des sciences sociales de Grenoble.

- L'animation de la recherche et de l'économie des régions, par Pierre Cognard, président de l'Alliance universitaire de Grenoble. 


\section{NOTES}

1.S. Bia, « Mathieu Joanicot, manager du futur », Le Journal du CNRS, 268, janvier 2004, p. 14.

2.Entretien avec François Juillet réalisé à Lyon le 24 novembre 2003.

3.Ibid.

4.Ibid.

5.Je fais ici référence aux tensions entre militaires, ingénieurs et chercheurs quant à la propriété du premier calculateur électronique appelé Eniac aux États-Unis dans l'immédiat après-guerre et au conflit qui opposa scientifiques et militaires, toujours aux États-Unis, à propos du secret des recherches atomiques.

6.Les travaux sur la recherche ont eu une importance considérable dans l'accélération du changement, d'autant plus qu'ils permettaient de mener une comparaison avec ce qui se faisait à l'étranger, en particulier aux États-Unis. Pour une analyse de ces travaux, voir G. Ramunni, Les Sciences pour l'ingénieur. Histoire du rendez-vous des sciences et de la société, Paris, CNRS ÉDITIONS, 1995.

7. « Un nouveau départ pour la recherche, une nouvelle organisation pour le CNRS », hors-série du Courrier du CNRS, 19, Paris, 1976.

8.Ibid.

9.Ibid.

10.Ibid.

11.Ibid.

12.Ibid.

13.D'après les statistiques de la DGRST en 1975, le personnel de la recherche, hors enseignants-chercheurs, était de 45 500, dont 15000 chercheurs et 30500 ITA.

14.Souhait du Comité interministériel de la recherche scientifique de février 1974. 15.Conseil restreint consacré à la recherche scientifique et technique de février 1975. 16.Archives DGRST, rapport du groupe « Politique de localisation de la recherche », mars 1976.

17.Ibid.

18.Inspection générale de l'administration, « Rapport sur le fonctionnement des administrations déléguées du CNRS », Paris, juin 1977.

19.Ibid.

20.Ibid.

21.Loi 82-213 du 2 mars 1982.

\section{RÉSUMÉS}

The Regionalization: Move Closer Research and Regional Realities

In 1975, the CNRS and Rhône-Poulenc, one of the leading chemical group located in Lyon, signed an agreement intended to share information and cooperate in some research programs. This 
agreement was prepared during the regular meetings organized by the representative of the DGRST. This new figure in the French administration was part of the experiment in order to test the possibilities of developing research activities outside Paris. The politics of relocation of scientific research centres, both in the basic research as in industrial research, was a priority in the second half of the seventies.

INDEX

Mots-clés : régionalisation, région

\section{AUTEUR}

\section{GIROLAMO RAMUNNI}

Girolamo Ramunni est professeur d'histoire des sciences à l'université de Lyon II. Il est le rédacteur en chef de La Revue pour l'histoire du CNRS. Il est responsable de l'action concertée incitative «Cultures et sociétés dans le développement durable » auprès du ministère de la Recherche et des Nouvelles Technologies. 